\title{
Ground glass opacities of the lung before, during and post COVID-19 pandemic
}

\author{
Marcello Migliore ${ }^{1,2} \wedge$ \\ ${ }^{1}$ Thoracic Surgery, Cardio-thoracic Department, University Hospital of Wales, Cardiff, UK; ${ }^{2}$ Thoracic Surgery, Department of General Surgery and \\ Medical Specialties, University of Catania, Catania, Italy \\ Correspondence to: Marcello Migliore, MD, PhD, FETCS. Thoracic Surgery, Cardio-thoracic Department, University Hospital of Wales, Cardiff, UK; \\ Thoracic Surgery, Department of General Surgery and Medical Specialties, University of Catania, Catania, Italy. Email: mmiglior@hotmail.com.
}

Submitted Apr 25, 2021. Accepted for publication May 23, 2021.

doi: 10.21037/atm-21-2095

View this article at: http://dx.doi.org/10.21037/atm-21-2095

The presence of ground glass lung opacity (GGO) in high resolution CT scan has become a routine part of thoracic surgical practice as demonstrated by the increase number of publications reported in PubMed. On the $16^{\text {th }}$ of March 2021, the following words have been searched "ground glass opacity and GGO", and 252 manuscripts were published in 2020.

It is a privilege that the Editor of Annals of Translational Medicine asked me to write this editorial commentary on the interesting paper of Wang et al. from West China Hospital of Sichuan University entitled "Distinct clinicopathologic factors and prognosis based on the presence of groundglass opacity components in patients with resected stage I non-small cell lung cancer" (1). The invitation provides an opportunity to glance at the fast-evolving picture of GGOs during COVID-19 Pandemic which makes the clinical scenario of GGOs in cancer patients far more complicated.

This commentary will therefore not only discuss the manuscript (1) but emphasize the challenges that thoracic surgeons, physicians and oncologists are now facing, and will face in future with GGOs during and post COVID-19 pandemic.

\section{GGOs challenges before the COVID-19 Pandemic}

The reader should know that the cohort of patients in the study (1) has been enrolled between 2009 and 2016 and therefore it is the ideal paper to discuss the challenges of GGOs in the pre COVID-19 pandemic era.
Although it is a very large study including 2,775 patients with a postoperative follow-up of more than 80 months, the study presents one unresolvable weakness: the retrospective nature of the paper generates missed data resulting in an inferior level of evidence, and consequentially the results must be decoded with caution.

However, the authors made an important contribution adding to our knowledge that GGO component was a predictor of favorable prognosis in stage I NSCLC patients. The next edition of tumor-node-metastasis (TNM) classification should consider the importance of GGO components as a new $\mathrm{T}$ descriptor. This suggestion was raised because the presence of the pure GGO in stage I lung cancer was significantly associated with the survival outcomes above $90 \%$ at 5 years follow-up.

I disagree with the Authors opinion that "pure-GGOs are relatively simple to assess". On the contrary, from my point of view the assessment could be difficult. For example, a hilar GGO represents a challenge for the surgeons as it could be difficult to obtain a preoperative diagnosis, and the surgical treatment will be more intriguing as the limits of the segments in some circumstances can be crowed, and therefore a $2 \mathrm{~cm}$ hilar GGO can easily involve 2 segments. Virtual bronchoscopy, 3D reconstruction and EBUS could be useful diagnostic tools in this situation as 20 of $31(65 \%)$ of pure GGO were successfully diagnosed in a recent study (2). Furthermore, the absence of preoperative diagnosis which is not uncommon in patients with GGOs, constitutes a challenge to the surgeon who need to follow a

\footnotetext{
^ ORCID: 0000-0002-6272-8983.
} 
three-step approach: (I) the lesion should first be localized; (II) the biopsy (wedge) for frozen section analysis should be performed and (III) if the histology is positive for malignancy the lung resection undertaken. Most of the time it is impossible to identify GGO during VATS even in the periphery of the lung, and thoracotomy could be necessary as demonstrated by Suzuki et al. (3) who reported $54 \%$ failure to visualize or palpate the nodule by VATS. More sophisticated diagnostic techniques could be used for intraoperative localization, but these are available only in specialized centers $(4,5)$.

The measure of long-term outcome is the most important end-point in cancer surgery, and the Authors demonstrated that "the presence of GGO components was a significant prognosticator (HR 0.415, 95\% CI: $0.286-$ 0.601). This exceptional long-term result was reported when only one GGO lung cancer has been removed. No data have been showed in case of a simultaneous second or third GGO in a different lobe or lung. It is evident that the Authors confirmed that lung cancer patients with pureGGOs have an extraordinary survival. Nevertheless, it is not possible to conclude that this long-term survival correlates with the stage or with the type of resection. Although data on lymphadenectomy were not reported, a previous study of 768 GGOs patients showed that lymph node metastasis was $0 \%$ in the GGO group. Whereas it was $3.8 \%$ and $6.9 \%$ in the mixed type and the predominantly solid group respectively. It seems therefore unnecessary to perform lymphadenectomy for patients with pure GGO in view of the $0 \%$ lymph node metastasis rate (6).

\section{GGOs during the COVID-19 Pandemic}

Let us now leave the GGOs in the pre COVID-era aside and consider the complexity of GGOs during the COVID-19 pandemic. Since the beginning of the COVID-19 Pandemic it has been shown that most patients present pneumonia typical imaging features, such as GGO, mixed GGO, vascular enlargement in the lesion, and consolidation. This data has been confirmed in the most cited articles reporting pneumonia secondary to COVID-19 where the presence of GGOs reaches $86 \%$ (7-10).

The identification of a single GGO on the initial CT scan suggests an early-phase of the COVID-19, it is then crucial to detect GGO as soon as possible in order to allow a proper evaluation thus improving early decision making and outcome.

The obvious question is do we need to differentiate a
GGO due to lung cancer from that related to COVID-19 during the COVID-19 Pandemic. My personal opinion is that during the acute phase of COVID-19, the need for early identification of GGOs is mandatory. However, the differential diagnosis with lung cancer it is not compulsory or albeit useful for three main reasons. The first is the fact that the GGO commonly tend to deteriorate quickly in COVID-19 patients causing the need for rapid hospitalization and oxygen therapy. The second reason relates to our understanding that the survival rate in GGO lung cancer surpass $90 \%$ at 5 years. The third would be our knowledge that lung cancer patients with COVID-19 have high in-hospital mortality (11), thus surgery could be safely deferred.

\section{GGOs post COVID-19 pandemic}

One might think that once the COVID-19 pneumonia heals, cancer diagnosis of GGO will resume its priority. I believe the future of GGO diagnosis and treatment after the COVID Pandemic will certainly become more complex.

Persistent symptoms which do not require further hospitalization have been defined as post-acute COVID-19 syndrome or 'long COVID' (12). It has been recently demonstrated that at 6 months follow-up a GGO is the most common pattern seen by high resolution CT scan. Irregular line is the second most common pattern (13). These GGOs are present in $41-49 \%$ of patients with long COVID at 6 months follow-up. The difficulties in the management approach of patients with residual GGO arise from a multitude of ambiguities. Will these GGOs disappear in the next few years? Will they transform to cancer? For example, is a single residual GGO after the resolution of COVID-19 pneumonia a cancer or a scar? It will become even more complicated in the presence of multiple residual GGOs. Are they all COVID 19 related or early coexisting lung cancer?

Unfortunately, high-resolution CT seems to be not helpful. Localized lesion, vacuoles, pleural traction, and invasion of pulmonary capillaries can be found in both COVID-19 and lung cancer. CT-PET needs further evaluation. A diagnosis would require a detailed medical history including smoking habits, routine blood tests, IgG and IgM blood levels, and pharyngeal swab.

Evaluation of CT scans for GGOs in terms of numbers, preexisting or new must be reported in order to reach a proper diagnosis who should assist in avoiding unnecessary lung cancer operations. If in doubts an asymptomatic 
patient with GGO should undergo a routine bronchoscopy with swab test taken from the lower respiratory tract to establish a diagnosis.

\section{Dedicated TNM classification for GGOs}

The Authors believe that their study provides evidence that the next edition of TNM classification should lay more importance on GGO as a new $\mathrm{T}$ descriptor (1). Several issues remain. The TNM classification of tumors is evolving continuously and interested specialists ask for further modifications based on innovations obtained by powerful prospective studies $(14,15)$. For example, I proposed a dedicated staging system for lung metastases $(16,17)$. It relies on the number of metastases and location of lymph nodes involved. This should provide a standardized nomenclature. For the same reasons, I agree with the Authors that a dedicated T for GGOs should be initiated within the TNM staging system. Perhaps, a simple prefix of "g or ggo" to the TNM (gTNM or ggoTNM) could be enough to distinguish it from solid lung tumors. In addition, a staging system for GGOs on the basis of diameter, the solid component, and the ratio between the different component could be developed.

\section{Conclusions}

There are many factors and complexities that should be taken into consideration in patients with GGO in the COVID-19 and post COVID-19 era. A standardized GGO staging nomenclature should be established to distinguish pure from mixed and solid GGOs within the TNM staging system, and this should have an international agreement.

\section{Acknowledgments}

I would like to thank Mr. Ayad Al-Bermani, Consultant Ophthalmologist, University Hospital of Wales, Cardiff, UK for his support and advice.

Funding: None.

\section{Footnote}

Provenance and Peer Review: This article was commissioned by the editorial office, Annals of Translational Medicine. The article did not undergo external peer review.

Conflicts of Interest: The author has completed the ICMJE uniform disclosure form (available at http://dx.doi. org/10.21037/atm-21-2095), and has no conflicts of interest to declare.

Ethical Statement: The author is accountable for all aspects of the work in ensuring that questions related to the accuracy or integrity of any part of the work are appropriately investigated and resolved.

Open Access Statement: This is an Open Access article distributed in accordance with the Creative Commons Attribution-NonCommercial-NoDerivs 4.0 International License (CC BY-NC-ND 4.0), which permits the noncommercial replication and distribution of the article with the strict proviso that no changes or edits are made and the original work is properly cited (including links to both the formal publication through the relevant DOI and the license). See: https://creativecommons.org/licenses/by-nc-nd/4.0/.

\section{References}

1. Wang C, Wu Y, Li J, et al. Distinct clinicopathologic factors and prognosis based on the presence of groundglass opacity components in patients with resected stage I non-small cell lung cancer. Ann Transl Med 2020;8:1133.

2. Piao Z, Han SJ, Cho HJ, et al. Feasibility of electromagnetic navigation bronchoscopy-guided lung resection for pulmonary ground-glass opacity nodules. J Thorac Dis 2020;12:2467-73.

3. Suzuki K, Nagai K, Yoshida J, et al. Video-assisted thoracoscopic surgery for small indeterminate pulmonary nodules: indications for preoperative marking. Chest 1999;115:563-8.

4. Nardini M, Bilancia R, Paul I, et al. 99mTechnetium and methylene blue guided pulmonary nodules resections: preliminary British experience. J Thorac Dis 2018;10:1015-21.

5. Migliore M, Fornito M, Palazzolo M, et al. Ground glass opacities management in the lung cancer screening era. Ann Transl Med 2018;6:90.

6. Lin YH, Chen CK, Hsieh CC, et al. Lymphadenectomy is Unnecessary for Pure Ground-Glass Opacity Pulmonary Nodules. J Clin Med 2020;9:672.

7. Zhou S, Wang Y, Zhu T, et al. CT Features of Coronavirus Disease 2019 (COVID-19) Pneumonia in 62 Patients in Wuhan, China. AJR Am J Roentgenol 2020;214:1287-94.

8. Fang Y, Zhang H, Xie J, et al. Sensitivity of Chest CT for COVID-19: Comparison to RT-PCR. Radiology 
2020;296:E115-7.

9. Zhao W, Zhong Z, Xie X, et al. Relation Between Chest CT Findings and Clinical Conditions of Coronavirus Disease (COVID-19) Pneumonia: A Multicenter Study. AJR Am J Roentgenol 2020;214:1072-7.

10. Zhu J, Zhang Y, Gao XH, et al. Coronavirus Disease 2019 or Lung Cancer: A Differential Diagnostic Experience and Management Model From Wuhan. J Thorac Oncol 2020;15:e141-2.

11. Nie L, Dai K, Wu J, et al. Clinical characteristics and risk factors for in-hospital mortality of lung cancer patients with COVID-19: A multicenter, retrospective, cohort study. Thorac Cancer 2021;12:57-65.

12. Greenhalgh T, Knight M, A'Court C, et al. Management of post-acute covid-19 in primary care. BMJ 2020;370:m3026.

13. Huang C, Huang L, Wang Y, et al. 6-month consequences of COVID-19 in patients discharged from hospital: a

Cite this article as: Migliore M. Ground glass opacities of the lung before, during and post COVID-19 pandemic. Ann Transl Med 2021;9(13):1042. doi: 10.21037/atm-21-2095 cohort study. Lancet 2021;397:220-32.

14. Rami-Porta R, Bolejack V, Crowley J, et al. The IASLC Lung Cancer Staging Project: Proposals for the Revisions of the T Descriptors in the Forthcoming Eighth Edition of the TNM Classification for Lung Cancer. J Thorac Oncol 2015;10:990-1003.

15. Aokage K, Miyoshi T, Ishii G, et al. Clinical and pathological staging validation in the eighth edition of the TNM classification for lung cancer: correlation between solid size on thin-Section computed tomography and invasive size in pathological findings in the new $\mathrm{T}$ classification. J Thorac Oncol 2017;12:1403-12.

16. Migliore M, Gonzalez M. Looking forward lung metastasectomy-do we need a staging system for lung metastases?. Ann Transl Med 2016;4:124.

17. Migliore M, Gonzalez M. TNM classification for lung metastases. Video-assist Thorac Surg 2021. [Epub ahead of print]. doi: 10.21037/vats-2020-lm-11. 